\title{
Mycoviruses in Fusarium Species: An Update
}

\section{Pengfei Li ${ }^{\dagger}$, Pallab Bhattacharjee ${ }^{\dagger}$, Shuangchao Wang, Lihang Zhang, Irfan Ahmed and Lihua Guo*}

State Key Laboratory for Biology of Plant Diseases and Insect Pests, Institute of Plant Protection, Chinese Academy of Agricultural Sciences, Beijing, China

\section{OPEN ACCESS}

Edited by: Daohong Jiang,

Huazhong Agricultural

University, China

Reviewed by:

Mingde Wu,

Huazhong Agricultural

University, China

Maria A. Ayllón

Polytechnic University of

Madrid, Spain

*Correspondence:

Lihua Guo

guolihua72@yahoo.com

†These authors have contributed equally to this work

Specialty section: This article was submitted to

Fungal Pathogenesis,

a section of the journal

Frontiers in Cellular and Infection

Microbiology

Received: 28 February 2019 Accepted: 03 July 2019 Published: 18 July 2019

Citation:

Li P, Bhattacharjee P, Wang S,

Zhang L, Ahmed I and Guo L (2019)

Mycoviruses in Fusarium Species:

An Update

Front. Cell. Infect. Microbiol. 9:257.

doi: 10.3389/fcimb.2019.00257
Fusarium is an important genus of plant pathogenic fungi, and is widely distributed in soil and associated with plants worldwide. The diversity of mycoviruses in Fusarium is increasing continuously due to the development and extensive use of state-of-the-art RNA deep sequencing techniques. To date, fully-sequenced mycoviruses have been reported in 13 Fusarium species: Fusarium asiaticum, F. boothii, F. circinatum, F. coeruleum, F. globosum, F. graminearum, F. incarnatum, F. langsethiae, F. oxysporum, F. poae, F. pseudograminearum, F. solani, and F. virguliforme. Most Fusarium mycoviruses establish latent infections, but some mycoviruses such as Fusarium graminearum virus 1 (FgV1), Fusarium graminearum virus-ch9 (FgV-ch9), Fusarium graminearum hypovirus 2 (FgHV2), and Fusarium oxysporum f. sp. dianthi mycovirus 1 (FodV1) cause hypovirulence. Rapid advances in various omics technologies used to elucidate genes or biological processes can facilitate an improved understanding of mycovirus-host interactions. The review aims to illuminate the recent advances in studies of mycoviruses in Fusarium, including those related to diversity, molecular mechanisms of virus-host interaction. We also discuss the induction and suppression of RNA silencing including the role of RNAi components as an antiviral defense response.

Keywords: Fusarium, mycovirus, mycovirus diversity, hypovirulence, mycovirus-host interactions, RNAi component

\section{INTRODUCTION}

According to the tenth report of the International Committee for Taxonomy of Viruses (ICTV), fungal viruses or mycoviruses have been classified into seven linear dsRNA virus families and one linear dsRNA virus genus (Chrysoviridae, Endornaviridae, Megabirnaviridae, Quadriviridae, Partitiviridae, Reoviridae, Totiviridae, and Botybirnavirus, respectively), six linear positive-sense ssRNA virus families (Alphaflexiviridae, Barnaviridae, Deltaflexiviridae, Gammaflexiviridae, Hypoviridae, and Narnaviridae), one linear negative-sense ssRNA virus family (Mymonaviridae), two reverse transcribing linear ssRNA virus families (Metaviridae and Pseudoviridae), and one circular ssDNA virus family (Genomoviridae) (Lefkowitz et al., 2018). No dsDNA mycoviruses have been isolated from fungi. 
Mycoviruses are generally transmitted vertically via spores and horizontally via anastomosis, with one exception, Sclerotinia gemycircularvirus 1 (formerly named Sclerotinia sclerotiorum hypovirulence-associated DNA virus 1), which can extracellularly infect fungi (Yu et al., 2013). Although many mycoviruses show latent infections, some cause phenotypic alterations that reduce or increase the virulence of the host (hypoor hypervirulence) (Nuss, 2005; Pearson et al., 2010; Jiang et al., 2013; Ghabrial et al., 2015). Fungal viruses causing hypovirulence attract much attention as potential candidates for the biological control of phytopathogenic fungal diseases and provide an opportunity to understand the interactions between mycoviruses and plant-pathogenic fungal hosts (Nuss, 2005; Xie and Jiang, 2014; Ghabrial et al., 2015). Because most viruses lack an extracellular replication phase, researchers have developed many transformation and transfection techniques using cDNA infectious clones (Choi and Nuss, 1992; Chen et al., 1993; Zhang et al., 2016), dsRNA (Stanway and Buck, 1984; Kanhayuwa et al., 2015), in vitro-transcribed RNA transcripts (Chen et al., 1994; Chen and Nuss, 1999; Moleleki et al., 2003; Lin et al., 2007; Marzano et al., 2015), and purified virions (Hillman et al., 2004; Sasaki et al., 2007). These techniques have accelerated the identification of host and/or viral factor(s) involved in the interactions between mycoviruses and their hosts (Son et al., 2015). These methods can also be used to extend the experimental host ranges of some mycoviruses, without the restriction of fungal vegetative incompatibility (Son et al., 2015).

Fusarium is a widely dispersed filamentous phytopathogenic fungus genus of the phylum Ascomycota, which causes serious damage to many field, ornamental, forest, and horticultural crops (Sharma et al., 2018). Many Fusarium species also produce mycotoxins such as trichothecenes and fumonisins that cause health problems in humans and livestock, and some of the greatest economic impacts are associated with deoxynivalenol (DON) and its derivatives (Munkvold, 2017). Many species of Fusarium also act as hosts for mycoviruses. Although many mycoviruses have been reported from different species of Fusarium, only a few isolates have a hypovirulent effect on their host, which is one of the major challenges to using mycoviruses efficiently as a biocontrol agent against Fusarium species. This review discusses recent advances in research related to mycoviruses infecting Fusarium species.

\section{DIVERSE VIRUSES IN FUSARIUM}

Due to the advancement and widespread use of next generation sequencing (NGS) techniques, knowledge of the diversity of known mycoviruses has rapidly increased in the past few years. To date, complete genome sequences are available for 29 mycoviruses, which have been identified from different species of Fusarium (Table 1), including 17 dsRNA viruses from six established or provisionally designated families (Crysoviridae, Megabirnaviridae, Partitiviridae, Totiviridae, Alternaviridae, Fusagraviridae) and one unassigned dsRNA group, 11 (+)ssRNA viruses from five established or provisionally designated families (Deltaflexiviridae, Hypoviridae, Narnaviridae, Fusariviridae, and
Tymoviridae) and one unassigned (+)ssRNA group, and one (-)ssRNA virus from the family Mymonaviridae.

\section{Double-Stranded RNA Mycoviruses Family Crysoviridae}

Three members of the family Crysoviridae have been isolated from Fusarium, Fusarium graminearum virus-ch9 (FgV-ch9) from F. graminearum strain China 9 (Darissa et al., 2011, 2012), Fusarium graminearum virus 2 (FgV2) from F. graminearum strain 98-8-60 (Chu et al., 2004; Yu et al., 2011), and Fusarium oxysporum $\mathrm{f}$. sp. dianthi mycovirus 1 (FodV1) from F. oxysporum f. sp. dianthi strain 116 (Lemus-Minor et al., 2015). The genome of FgV-ch9 consists of five dsRNAs, denoted as dsRNA1 to dsRNA5, 3,581, 2,850, 2,830, 2,746, and 2,423 bp in size, respectively, each containing a single open reading frame (ORF). These five dsRNAs are encapsidated separately in unequal amounts. Among those five dsRNAs, dsRNA1 encodes the viral RNA-dependent RNA polymerase (RdRp), $127 \mathrm{kDa}$ in size, dsRNA2, and dsRNA3 encode two structural proteins, 94 and $93 \mathrm{kDa}$, resptectively. FgV-ch9 dsRNA4 and dsRNA5 encode polypeptides of unknown function, 91 and $79 \mathrm{kDa}$ in size, respectively. The FgV2 genome also consists of five dsRNA segments referred to as dsRNA1 to dsRNA5, 3,580, 3,000, 2,982, 2,748 , and $2,414 \mathrm{bp}$ in size, respectively, each containing a single ORF flanked by conserved $5^{\prime}$ and $3^{\prime}$ untranslated regions (UTRs). FgV2 dsRNA1 encodes the viral RdRp, which is 127 $\mathrm{kDa}$ in size, while the other four dsRNAs encode proteins of unknown function ( $\mathrm{Yu}$ et al., 2011). The FodV1 genome contains four dsRNA segments, 3,555, 2,809, 2,794, and 2,646 bp in length, respectively. Each of these segments contains a single ORF. FodV1 dsRNA1 and dsRNA3 encode an RdRp and a coat protein (CP), respectively, while dsRNA2 and dsRNA4 encode hypothetical proteins (named P2 and P4) with unknown functions (Lemus-Minor et al., 2015).

\section{Family Megabirnaviridae}

A member of the family Megabirnaviridae has been isolated from Fusarium pseudograminearum strain FC136-2A and is designated Fusarium pseudograminearum megabirnavirus 1 (FpgMBV1) (Zhang et al., 2018). The genome of FpgMBV1 consists of two dsRNA segments, L1-dsRNA (8,951 bp), and L2-dsRNA (5,337 bp), each containing two ORFs. L1-dsRNA encodes a CP of $131 \mathrm{kDa}$ and an RdRp of $126 \mathrm{kDa}$, while L2-dsRNA encodes two proteins of $97 \mathrm{kDa}$ and $31 \mathrm{kDa}$ with unknown functions.

\section{Family Partitiviridae}

Three members of the family Partitiviridae have been isolated from Fusarium, Fusarium solani virus 1 (FsV1; synonym, FusoV) from Fusarium solani f. sp. rohiniae strain SUF704 (Nogawa et al., 1996), Fusarium poae virus 1 (FpV1; synonym, FuPO-1) from Fusarium poae strain A-11 (Compel et al., 1999), and Fusarium solani partitivirus 2 (FsPV2) from Fusarium solani f. sp. pisi (Osaki et al., 2015). The FsV1 genome consists of two dsRNAs, 1,645 and 1,445 bp in size, each containing one ORF. The large dsRNA encodes the viral RdRp, $60 \mathrm{kDa}$ in size, while the small dsRNA encodes the CP, $44 \mathrm{kDa}$ in size (Nogawa et al., 1996). The 
TABLE 1 | Fully-sequenced Fusarium-infecting mycoviruses.

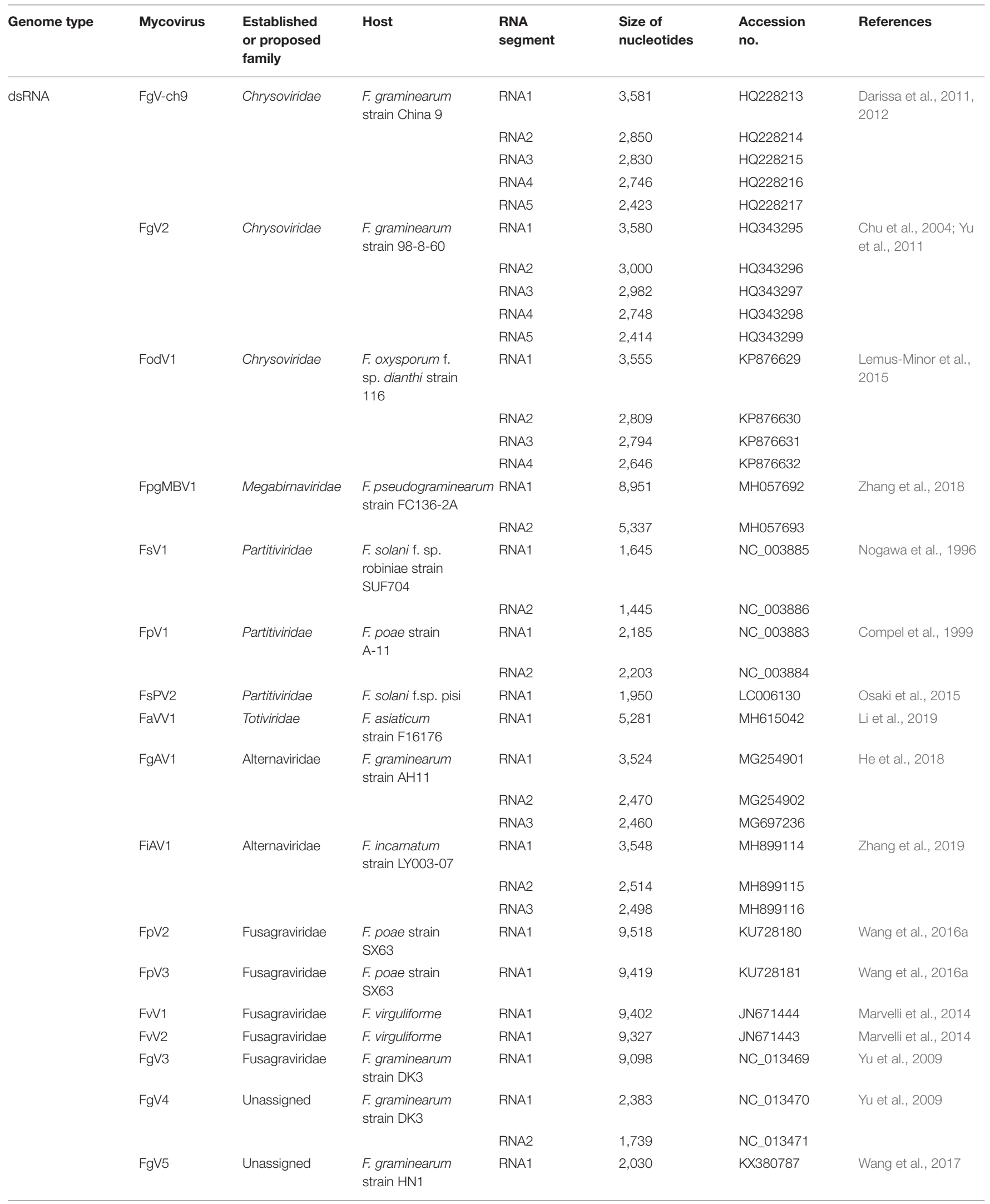


TABLE 1 | Continued

\begin{tabular}{|c|c|c|c|c|c|c|c|}
\hline Genome type & Mycovirus & $\begin{array}{l}\text { Established } \\
\text { or proposed } \\
\text { family }\end{array}$ & Host & $\begin{array}{l}\text { RNA } \\
\text { segment }\end{array}$ & $\begin{array}{l}\text { Size of } \\
\text { nucleotides }\end{array}$ & $\begin{array}{l}\text { Accession } \\
\text { no. }\end{array}$ & References \\
\hline & & & & RNA2 & 1,740 & KX380788 & \\
\hline \multirow[t]{13}{*}{$(+)$ ssRNA } & FgDFV1 & Deltaflexiviridae & $\begin{array}{l}\text { F. graminearum } \\
\text { strain BJ59 }\end{array}$ & RNA1 & 8,246 & KX015962 & Chen et al., 2016 \\
\hline & FgHV1 & Hypoviridae & $\begin{array}{l}\text { F. graminearum } \\
\text { strain HN10 }\end{array}$ & RNA1 & 13,023 & KC330231 & Wang et al., 2013 \\
\hline & $\mathrm{FgHV} 2$ & Hypoviridae & $\begin{array}{l}\text { F. graminearum } \\
\text { strain JS16 }\end{array}$ & RNA1 & 12,800 & KP208178 & Li et al., 2015 \\
\hline & $\mathrm{FlHV} 1$ & Hypoviridae & $\begin{array}{l}\text { F. langsethiae } \\
\text { strain } \mathrm{AH} 32\end{array}$ & RNA1 & 12,839 & KY120321 & Li et al., 2017 \\
\hline & FCMV1 & Narnaviridae & $\begin{array}{l}\text { F. circinatum } \\
\text { strain FcCa070 }\end{array}$ & RNA1 & 2,419 & KF803546 & $\begin{array}{l}\text { Martínez-Álvarez } \\
\text { et al., } 2014\end{array}$ \\
\hline & FcoMV1 & Narnaviridae & $\begin{array}{l}\text { F. coeruleum } \\
\text { (MAFF No. } \\
\text { 235976) }\end{array}$ & RNA1 & 2,423 & LC006129 & Osaki et al., 2015 \\
\hline & FgMV1 & Narnaviridae & $\begin{array}{l}\text { F. globosum } \\
\text { (MAFF No. } \\
237511 \text { ) }\end{array}$ & RNA1 & 2,414 & LC006128 & Osaki et al., 2015 \\
\hline & FbMV1 & Narnaviridae & $\begin{array}{l}\text { F. boothii strain } \\
\text { Ep-BL13 }\end{array}$ & RNA1 & 2,802 & LC425112 & Mizutani et al., 2018 \\
\hline & & Narnaviridae & $\begin{array}{l}\text { F. boothii strain } \\
\text { Ep-BL14 }\end{array}$ & RNA1 & 2,801 & LC425113 & \\
\hline & & Narnaviridae & $\begin{array}{l}\text { F. boothii strain } \\
\text { Ep-N28 }\end{array}$ & RNA1 & 2,802 & LC425114 & \\
\hline & FgV1 & Fusariviridae & $\begin{array}{l}\text { F. graminearum } \\
\text { strain DK21 }\end{array}$ & RNA1 & 6,624 & NC_006937 & $\begin{array}{l}\text { Chu et al., 2002; } \\
\text { Kwon et al., } 2007\end{array}$ \\
\hline & FgMTV1 & Tymoviridae & $\begin{array}{l}\text { F. graminearum } \\
\text { strain SX64 }\end{array}$ & RNA1 & 7,863 & KT360947 & Li et al., 2016 \\
\hline & FbLFV1 & Unassigned & $\begin{array}{l}\text { F. boothii strain } \\
\text { Ep-BL13 }\end{array}$ & RNA1 & 12,579 & LC425115 & Mizutani et al., 2018 \\
\hline$(-)$ ssRNA & FgNSRV-1 & Mymonaviridae & $\begin{array}{l}\text { F. graminearum } \\
\text { strain HN1 }\end{array}$ & RNA1 & 9,072 & MF276904 & Wang et al., 2018 \\
\hline
\end{tabular}

FpV1 genome consists of two dsRNAs, 2,185 and 2,203 bp in size. The two dsRNAs encode the viral RdRp and CP, 70 and $74 \mathrm{kDa}$ in size, respectively (Compel et al., 1999). FsPV2 dsRNA1 (1,950 bp) in F. solani f. sp. pisi contains an ORF encoding an RdRp, $72 \mathrm{kDa}$ in size. Sequence and phylogenetic analysis of the viral RdRps show that FsPV2 belongs to the genus Alphapartitivirus, family Partitiviridae. In general, partitiviruses possess two essential dsRNA genome segments: dsRNA1 (encoding the RdRp), and dsRNA2 (encoding the CP). However, the attempt to clone dsRNA2 of FsPV2 has failed (Osaki et al., 2015).

\section{Family Totiviridae}

A member of the genus Victorivirus, family Totiviridae, has been isolated from Fusarium asiaticum strain F16176 and is designated Fusarium asiaticum victorivirus 1 (FaVV1) (Li et al., 2019). The genome of FaVV1 consists of a single, linear dsRNA of 5,281 bp that contains two ORFs. ORF1 is predicted to encode a CP with a molecular mass of $79 \mathrm{kDa}$. ORF2 is predicted to encode an RdRp with a molecular mass of $92 \mathrm{kDa}$. Interestingly, the $\mathrm{CP}$ and $\mathrm{RdRp}$ of FaVV1 are most similar (77 and 75\% identical, respectively) to those of Rosellinia necatrix victorivirus 1 (RnVV1), which infects the white root rot pathogen fungus Rosellinia necatrix. Both ORFs overlap with each other at a pentanucleotide "UAAUG," and the overlapping pentanucleotide is one of the features of the genus Victorivirus (Li et al., 2019).

\section{Family Alternaviridae}

Two members of the newly proposed family Alternaviridae have been isolated from Fusarium, Fusarium graminearum alternavirus 1 (FgAV1) from F. graminearum strain AH11 (He et al., 2018), Fusarium incarnatum alternavirus 1 (FiAV1) from F. incarnatum strain LY003-07 (Zhang et al., 2019). The genome of FgAV1 possesses three dsRNA segments, dsRNA1 (3,524 bp), dsRNA2 (2,470 bp), and dsRNA3 (2,460 bp), each coding an ORF. FgAV1 dsRNA1 encodes an RdRp, $126 \mathrm{kDa}$ in size, while dsRNA2 and dsRNA3 encode polypeptides of unknown function, 84 and $81 \mathrm{kDa}$ in size, respectively. The RdRp of FgAV1 is most similar to Fusarium poae alternavirus 1 (FpAV1), with 98\% (ORF1), 99\% (ORF2), and 98\% (ORF3) aa sequence identities (He et al., 2018), while the terminal sequences of FpAV1 have not been determined (Osaki et al., 2016). Compared to the genomic composition (4 dsRNA segments) of three other members of 
this family, namely, Alternaria alternata virus 1 (AaV1) (Aoki et al., 2009), Aspergillus mycovirus 341 (AMV) (Hammond et al., 2008), and Aspergillus foetidus mycovirus (AfMV) (Kozlakidis et al., 2013), FgAV1/AH11 and FpAV1 both lack the fourth segment. The genome of FiAV1 also consists of three dsRNA segments, dsRNA1 (3,548 bp), dsRNA2 (2,514 bp), and dsRNA3 (2,498 bp), each encoding a single ORF. FiAV1 dsRNA1 encodes an RdRp of $127 \mathrm{kDa}$, while dsRNA2 and dsRNA3 encode proteins with unknown functions (Zhang et al., 2019).

\section{Family Fusagraviridae}

Five members of the newly proposed family Fusagraviridae (Wang et al., 2016a) have been isolated from Fusarium, Fusarium poae dsRNA virus 2 (FpV2), and Fusarium poae dsRNA virus 3 (FpV3) isolated from the same Fusarium poae strain SX63 (Wang et al., 2016a), Fusarium virguliforme dsRNA mycovirus 1 (FvV1) and Fusarium virguliforme dsRNA mycovirus 2 (FvV2) isolated from Fusarium virguliforme (Marvelli et al., 2014), and Fusarium graminearum virus 3 ( FgV3) isolated from $F$. graminearum strain DK3 (Yu et al., 2009). The respective genomes of FpV2 and FpV3 consist of a single dsRNA, 9,518 and 9,419 bp, respectively. Each contains two ORFs (ORF1 and ORF2), and encode a protein of unknown function and an RdRp, respectively (Wang et al., 2016a). A phytoreo_S7 domain is found downstream of the RdRp domain of the ORF2-coded proteins of both FpV2 and FpV3. The same shifty heptamer motif (GGAAAAC) is found immediately before the stop codon UAG of ORF1 in both FpV2 and $\mathrm{FpV} 3$, which could mediate programmed -1 ribosomal frameshifting ( $-1 \mathrm{PRF})$. The respective genomes of both $\mathrm{FvV1}$ and FvV2 consist of a dsRNA, 9,402 and 9,327 bp, respectively, and contain two ORFs (ORF1 and ORF2) that overlap by 25 nt. The 25-nt overlap between ORFs 1 and 2 contain a slippery sequence (AAAAAAC) that is a-1 frameshift signal. ORF2 encodes an RdRp, while the function of ORF1 is unclear (Marvelli et al., 2014). The FgV3 genome also has a dsRNA (9,098 bp), and possesses two ORFs. ORF1 encodes a protein of unknown function, while ORF2 encodes an RdRp. A phytoreovirus S7 protein domain is also found downstream of the $\operatorname{RdRp}$ domain of ORF2 (Yu et al., 2009).

\section{Unassigned dsRNA Mycoviruses in Fusarium}

Fusarium graminearum virus $4(\mathrm{FgV} 4)$ has been isolated from F. graminearum strain DK3 (Yu et al., 2009). The FgV4 genome consists of two dsRNAs, 2,383 and 1,739 bp, respectively. FgV4 dsRNA1 contains a single ORF encoding the viral RdRp, while FgV4 dsRNA2 contains two putative ORFs coding for products of unknown function. Fusarium graminearum dsRNA virus 5 (FgV5) has been isolated from Fusarium graminearum strain HN1 (Wang et al., 2017). The FgV5 genome comprises two dsRNAs (dsRNA1 and dsRNA2), 2,030 and 1,740 bp, respectively. FgV5 dsRNA1 contains an ORF encoding an RdRp, $70 \mathrm{kDa}$. FgV5 dsRNA2 contains two ORFs (ORF2 and ORF3) that code for products of unknown function. Phylogenetic analysis indicates that $\mathrm{FgV} 4$ and $\mathrm{FgV} 5$ belong to a taxonomically unassigned dsRNA mycovirus group that is related to the families Amalgaviridae and Partitiviridae (Wang et al., 2017).

\section{Positive-Sense, Single-Stranded RNA Mycoviruses \\ Family Deltaflexiviridae}

A member of the family Deltaflexiviridae has been isolated from F. graminearum strain BJ59 and is designated as Fusarium graminearum deltaflexivirus 1 (FgDFV1) (Chen et al., 2016). The FgDFV1 genome harbors a linear, positive-sense ssRNA, 8,246 nt in length excluding the poly (A) tails, and contains five putative ORFs. The largest ORF (ORF1) encodes a replication-associated polyprotein (RP) of $227 \mathrm{kDa}$, containing three conserved domains including viral RdRp, Hel, and Mtr. The four smaller ORFs (ORFs 2-5) encode four proteins of unknown function, 12, 13,18 , and $17 \mathrm{kDa}$, respectively.

\section{Family Hypoviridae}

Three members of the family Hypoviridae have been isolated from Fusarium, Fusarium graminearum hypovirus 1 (FgHV1) from Fusarium graminearum strain HN10 (Wang et al., 2013), FgHV2 from Fusarium graminearum strain JS16 (Li et al., 2015), and Fusarium langsethiae hypovirus 1 (FlHV1) from Fusarium langsethiae strain AH32 (Li et al., 2017). The FgHV1 genome consists of a linear, positive-sense ssRNA of 13,023 nt containing two ORFs (ORF A and ORF B). The $5^{\prime}$ proximal ORF A encodes a papain-like proteinase (p20), which is closely related to the Cryphonectria hypovirus 1 (CHV1)-encoded RNA silencing suppressor (RSS; p29) (Wang et al., 2016c). The $3^{\prime}$ proximal ORF $\mathrm{B}$ encodes a large polyprotein of $421 \mathrm{kDa}$ with three conserved domains including papain-like protease (Pro), RdRp, and RNA helicase (Hel) (Wang et al., 2013). The FgHV2 genome consists of a linear, positive-sense ssRNA of 12,800 nt in size, containing a single ORF. The ORF encodes a large polyprotein of $446 \mathrm{kDa}$ with three conserved domains, Pro, RdRp, and Hel, and a novel domain with homologous bacterial SMC (structural maintenance of chromosomes) chromosome segregation proteins (Li et al., 2015). The genome of FIHV1 contains a linear, positive-sense ssRNA, 12,839 nt in size, encoding a single ORF. The ORF encodes a polyprotein of $447 \mathrm{kDa}$, containing three conserved domains, Pro, RdRp, and Hel, which is a common feature of the family Hypoviridae.

\section{Family Narnaviridae}

Four members of the genus Mitovirus (family Narnaviridae) have been isolated from Fusarium, Fusarium circinatum mitovirus 1 (FcMV1) from Fusarium circinatum strain FcCa070 (MartínezÁlvarez et al., 2014), Fusarium coeruleum mitovirus 1 (FcoMV1) and Fusarium globosum mitovirus 1 (FgMV1) from Fusarium coeruleum (MAFF No. 235976) and Fusarium globosum (MAFF No. 237511), respectively (Osaki et al., 2015), and Fusarium boothii mitovirus 1 (FbMV1) from three different strains of Fusarium boothii: Ep-BL13, Ep-BL14, and Ep-N28 (Mizutani et al., 2018). The FcMV1 genome consists of a linear, positivesense ssRNA of 2,419 nt, containing a single ORF that encodes an RdRp of $85 \mathrm{kDa}$. The genomes of FcoMV1 and FgMV1 consist of linear, positive-sense ssRNA, 2,423 and 2,414 nt, respectively, each containing a single ORF encoding an RdRp (86 and 84 $\mathrm{kDa}$, respectively). FbMV1 genomes in strains Ep-BL13 and 
Ep-N28 are 2,802 nt in length and the genome in strain EpBL14 is 2,801 nt in length. These three virus strains share $98 \%$ nucleotide identity and each contains a single ORF encoding an RdRp.

\section{Family Fusariviridae}

A member of the newly proposed family, Fusariviridae has been isolated from F. graminearum strain DK21 (Chu et al., 2002; Zhang et al., 2014) and is designated Fusarium graminearum virus 1 strain DK21 (FgV1-DK21) (Yu et al., 2009). The genome of FgV1 consists of a linear, positive-sense ssRNA of 6,621 nt in length excluding the $3^{\prime}$-terminal poly (A) tail and contains four ORFs. The four ORFs (1-4) encode proteins with molecular masses of 174, 16.7, 6.2, $48.4 \mathrm{kDa}$, respectively, among which ORF1 encodes an RdRp, while the products of ORFs 2-4 reveal no obvious similarities to other viral sequences in the NCBI protein database (Kwon et al., 2007).

\section{Family Tymoviridae}

A member of the newly proposed genus Mycotymovirus, family Tymoviridae has been isolated from F. graminearum strain SX64 and is designated Fusarium graminearum mycotymovirus 1 (FgMTV1) (Li et al., 2016). The FgMTV1 genome consists of a linear, positive-sense ssRNA of 7,863 nt excluding the poly (A) tails, and contains four ORFs. ORF1 encodes a RP of $249 \mathrm{kDa}$, containing four conserved domains, viral RNA methyltransferase (Mtr), tymovirus endopeptidase (Pro), RdRp, and Hel. These four conserved domains are the common features of members of the family Tymoviridae (Martelli et al., 2002). ORFs 2-4 encode three hypothetical proteins of unknown function, 14, 13, and $19 \mathrm{kDa}$, respectively. FgMTV1 is the first tymo-like mycovirus isolated from a plant pathogenic fungus (Li et al., 2016).

\section{Unassigned (+)ssRNA Mycoviruses in Fusarium}

Fusarium boothii large flexivirus 1 (FbLFV1) has been isolated from Fusarium boothii strain Ep-BL13 (Mizutani et al., 2018). The FbLFV1 genome consists of a linear, positive-sense ssRNA of 12,579 nt, and encompasses a single large ORF encoding a replicase of $444 \mathrm{kDa}$, containing three conserved domains, Mtr, Hel, and RdRp. There are also two regions of unknown function in the polypeptide with similarity to a PHA03247 domain found in herpesviruses (large dsDNA viruses). Sequence and phylogenetic analyses show that FbLFV1 belongs to a novel virus species that may form an independent genus, or even a novel family, in the order Tymovirales (Mizutani et al., 2018).

\section{Negative-Sense, Single-Stranded RNA Mycoviruses \\ Family Mymonaviridae}

A member of the family Mymonaviridae has been isolated from Fusarium graminearum strain $\mathrm{HN} 1$ and is designated Fusarium graminearum negative-stranded RNA virus 1 (FgNSRV-1) (Wang et al., 2018). The genome of FgNSRV-1 harbors a linear, negative-sense ssRNA of 9,072 nt, and contains five discontinuous but linear ORFs (ORF I-V), encoding five proteins (termed P I-P V) with molecular masses ranging from 6 to 221 $\mathrm{kDa}$. Among the five FgNSRV-1 proteins, P II and P IV are related to the putative nucleoprotein $\mathrm{N}$ and large protein $\mathrm{L}$ of Sclerotinia sclerotiorum negative-stranded RNA virus 1 (SsNSRV-1) and SsNSRV-3 (Liu et al., 2014; Lee Marzano et al., 2016). The $3^{\prime}$ and $5^{\prime}$-ends of FgNSRV-1 RNA have perfect complementarity of the first six residues ( $3^{\prime}$-UCCUGC-GCAGGA-5'), which is a common feature among mononegaviruses (Chen et al., 2016).

\section{EFFECT OF VIRUSES ON FUSARIUM}

Due to recent technological advances, a number of virus/fungal host systems can be established by curing viruses along with artificial introduction (Kondo et al., 2013; Suzuki, 2017). The vegetative growth, development, and physiological properties of host fungi are highly influenced by the harmful, beneficial, or neutral (little or no) effects of fungal viruses (Kondo et al., 2013). In this review, we mainly focus on the interplay between mycoviruses and Fusarium spp.

Mitigation of fungal virulence or "hypovirulence" is a good example of the harmful effect of several viruses on their host fungi. Therefore, fungal viruses could act as a potential candidate for the biological control of fungi (Hillman et al., 2018). The most studied example of reduced fungal virulence is $\mathrm{CHV} 1 / \mathrm{C}$. parasitica (Dawe and Nuss, 2013). In the case of Fusarium spp., four mycoviruses have harmful effects on fungal phenotypes. FgHV2, a novel hypovirus of the newly proposed genus Alphahypovirus, is associated with hypovirulence phenotypes, including reduced mycelial growth rate, conidia production, and DON concentration. In the virulence assay, the virusfree strain spreads more quickly from the inoculation sites to nearby spikelets than the virus-carrying strain JS16 (Li et al., 2015). FgV1, a member of the novel family Fusariviridae, has been reported to reduce the virulence of $F$. graminearum, delay mycelial growth, increase pigmentation, and reduce the production of mycotoxin (Chu et al., 2002). FgV-ch9, a member of the family Crysoviridae, is associated with hypovirulence of F. graminearum at high and medium viral dsRNA levels, including reduced mycelial growth rate and conidiation capacity, abnormal colony morphology, disorganized cytoplasm, and reduced virulence in wheat and maize plants (Darissa et al., 2012). FodV1, a member of the family Chrysoviridae, causes significant phenotypic alterations in the vegetative growth and virulence of the fungal host (Lemus-Minor et al., 2018).

In most cases, mycovirus infection causes little or no symptoms in the host (Ghabrial and Suzuki, 2009). For example, FgV3 and FgV4 infections do not cause any phenotypic change in the host F. graminearum (Lee et al., 2014). FgHV1 infection has mild or no effects on the fungal phenotypes of F. graminearum including morphology, mycelial growth, conidiation, and virulence or toxin production (Wang et al., 2013). FsV1 and FpV1 are also not associated with phenotypic changes in their hosts F. solani and F. poae (Nogawa et al., 1996; Compel et al., 1999). Another two mycoviruses, FpV2 and FpV3, isolated from the same strain of Fusarium poae, do not show any deleterious effect on their host fungus (Wang et al., 2016a). 


\section{GENE REGULATIONS ASSOCIATED WITH FUSARIUM VIRUS INFECTION}

The proteome and transcriptome analysis of virus-infected fungi will help to elucidate the proteins and genes regulated by mycovirus infection that are involved in growth, development, and stress responses and direct further studies on the interactions between pathogenic fungi and viruses. The differential expression of $F$. graminearum proteins caused by $\mathrm{FgV1}$ infection was investigated by two-dimensional electrophoresis with mass spectrometry (Kwon et al., 2009). Seven proteins, including sporulation-specific gene SPS2, triose phosphate isomerase, nucleoside diphosphate kinase, and woronin body major protein precursor, were induced or significantly upregulated by $\mathrm{FgV1}$ infection. The significant decrease or down regulation of 16 proteins, including enolase, saccharopine dehydrogenase, flavohemoglobin, mannitol dehydrogenase, and malate dehydrogenase, caused by $\mathrm{FgV1}$ infection was also identified. However, the number of proteins identified was insufficient to obtain a comprehensive understanding of the host response (Kwon et al., 2009; Cho et al., 2013). Therefore, genome-wide expression analyses were carried out using a $3^{\prime}$-tiling microarray in $\mathrm{FgV} 1$-infected F. graminearum. Genes associated with protein synthesis (such as ribosome assembly, nucleolus, and ribosomal RNA processing) and genes required for transcription and signal transduction (such as fungal-specific transcription factors and cAMP signaling), were significantly up-regulated in fungal host cells, which seems to be related to virus replication. In contrast, significant down-regulation of genes required for metabolism (such as carboxylic acids, aromatic amino acids, nitrogen compounds, and polyamines) and transporting systems in a fungal host containing the virus appears to be related to the host defense mechanism and fungal virulence (Cho et al., 2012, 2013). Furthermore, a comprehensive genome-wide gene expression analysis elucidated completely distinct expression patterns of $F$. graminearum transcriptomes in response to infections by two hypovirulent mycoviruses (FgV1 and $\mathrm{FgV} 2$ ) and two non hypovirulent mycoviruses ( $\mathrm{FgV} 3$ and $\mathrm{FgV} 4$ ), respectively. Interestingly, changes in the host transcriptome caused by different mycoviruses are not always correlated with observed host phenotypes. The fungal host transcriptome was more affected by $\mathrm{FgV} 1$ and $\mathrm{FgV} 4$ infections than by $\mathrm{FgV} 2$ and $\mathrm{FgV} 3$ infections. In addition, 12 differentially expressed genes were identified in response to all four mycovirus infections, but functions of most of these genes are still unknown (Lee et al., 2014). In the FgHV1-infected F. graminearum strain, enriched genes related to redox regulation are differentially expressed. It has been demonstrated that FgHV1-encoded p20 could induce $\mathrm{H}_{2} \mathrm{O}_{2}$ accumulation and a hypersensitive response in Nicotiana benthamiana leaves (Wang et al., 2016c).

Subsequently, the hexagonal peroxisome protein FgHex1 was screened using transcriptional and proteomic analyses. By generating FgHEX1 gene deletion and overexpression mutants, it was indicated that the FgHEX1 gene plays a direct role in the asexual reproduction and virulence of $F$. graminearum and facilitates viral RNA accumulation in the FgV1-infected host fungus (Son et al., 2013). To investigate the molecular mechanism underlying the production of FgHEX1 and the replication of FgV1 viral RNA, electrophoretic mobility shift assays (EMSA) was conducted with recombinant FgHexl protein and RNA sequences derived from various regions of $\mathrm{FgV1}$ genomic RNA. These analyses demonstrated that the FgHex1 functions in the synthesis of both strands of FgV1 RNA and therefore in $\mathrm{FgV1}$ replication probably by specifically binding the FgV1 genomic RNA. This is the first report about the regulation of viral RNA replication by a fungal cellular protein that can directly bind to viral genomic RNA (Son et al., 2016). Otherwise, another host gene, the putative $3^{\prime}\left(2^{\prime}\right), 5^{\prime}$-bisphosphate nucleotidase gene, FgHal2, in F. graminearum is down-regulated following FgV1 infection. The possible function(s) of FgHal2 was investigated in $F$. graminearum using gene deletion and gene over-expression mutants. It was found that deletion of $\mathrm{FgHal} 2$ reduced conidiation, mycelial growth, and the production of secondary metabolites. Moreover, deletion of FgHal2 decreased viral RNA accumulation and the vertical transmission of FgV1 via conidia. Together, these results indicate that $F$. graminearum can down-regulate one of its major multifunctional genes, $\mathrm{FgHal2}$, in response to FgV1 infection (Yu et al., 2015). Recently, an mRNA binding protein (named virus response 1, vrl) was identified to be involved in symptom expression in $F$. graminearum. Downregulation of $v r 1$ in the virus FgV-ch9 infected fungus and $v r 1$ deletion evoke virus-infection like symptoms while constitutive expression overrules the cytopathic effects of the virus infection. Intriguingly, the presence of a specific viral structural protein $\mathrm{P} 3$ is sufficient to trigger the fungal response, i.e., vrl downregulation, and symptom development similar to virus infection. Hence, $v r 1$ represents a fundamental host factor for the expression of virus-related symptoms and helps to understand the underlying mechanism of hypovirulence. The advancements in understanding fungal infection and response may aid biological pest control approaches using mycoviruses or viral proteins to prevent future Fusarium epidemics (Bormann et al., 2018).

\section{RNAI IN FUSARIUM RESPONDING TO VIRAL INFECTION}

RNA silencing or RNA interference (RNAi) functions as an antiviral defense mechanism in eukaryotic organisms. The core elements of the cross-kingdom RNA silencing defense response consists of conserved ribonucleases: members of the Dicerlike (DCL) and Argonaute-like (AGO) protein families. Dicer nucleases recognize viral double-stranded and structured RNAs and use the associated RNase III-type activity to process these RNAs into small RNAs of 21-24 nts in length, termed virusderived small (vs.) RNAs. The vsRNAs are then incorporated into an effector complex termed the RNA-induced silencing complex (RISC) with the aid of an Argonaute family protein. One strand of the vsRNA is degraded and the remaining guide strand targets the effector complex to the cognate viral RNA, which is then cleaved by the Argonaute-associated RNAse Hlike activity. Subsequently, the antiviral RNA silencing response is further amplified by host RNA-dependent RNA polymerases 
(RdRPs) (Ding, 2010; Nuss, 2011). To combat RNA silencingbased antiviral defense, viruses of plants, insects, mammals, and fungi encode proteins, designated viral suppressors of RNA silencing (VSR), that employ a variety of mechanisms to suppress RNA silencing pathways. RNA silencing and viruses against host RNA silencing have been well-characterized in the Cryphonectria parasitica-hypovirus system (Ding, 2010; Nuss, 2011). More encouragingly, the roles of core components of the RNAi pathway have been also been gradually revealed in F. graminearum.

F. graminearum contains genes encoding two dicer proteins (FgDicer1 and FgDicer2), two Argonaute proteins (FgAgo1 and FgAgo2), and five RdRp proteins (FgRdRp1 to 5). Chen et al. showed that RNAi machinery was not involved in growth, abiotic stress and pathogenesis in F. graminearum. In addition, it was demonstrated that the hairpin RNA (hpRNA) can efficiently silence the expression level of target gene and the argonaute protein FgAgol and dicer protein FgDicer2 played a critical role in the hpRNA mediated gene silencing process (Chen et al., 2015). FgDicer2 was also involved in small RNA (sRNA, 17-40 nt) transcription and microlike-RNA (milRNA) generation in this fungus (Chen et al., 2015). Strikingly, a recent study showed that FgDicer2 is the primary DCL component for defense against viroids in $F$. graminearum (Wei et al., 2019). Furthermore, a combined analysis of functional genetics and deep sequencing of small non-coding RNA (sRNA), mRNA, and the degradome indicate that the sex-specifically induced exonic small interference RNA (exsiRNA)-mediated RNA interference (RNAi) mechanism has an important role in fine-tuning the transcriptome during ascospore formation in F. graminearum. It is FgDicer1 and FgAgo2 that primarily mediate the sex-specific RNAi pathway. Each fungal species appears to have evolved RNAi-based gene regulation for specific developmental stages or stress responses. This study provides new insights into the regulatory role of sRNAs in fungi and other lower eukaryotes (Son et al., 2017).

To preliminarily elucidate the RNA silencing mechanism of the F. graminearum/hypovirus system, the properties of sRNAs in FgHV1 and FgHV2 were analyzed by using HiSeq deep sequencing in F. graminearum. The length distributions of F. graminearum sRNAs were altered by hypoviral infection. Potential milRNA candidates were differentially expressed between the hypovirus-free and hypovirus-infected library types. The 1,831,081 and 3,254,758 total reads generated from the FgHV1 and FgHV2 genomes in F. graminearum yielded the first high-resolution sRNA maps of fungal viruses. In particular, FgDicer1 and FgRdRp5 were predicted targets of FgHV1- and FgHV2-derived siRNAs, possibly revealing a novel anti-RNA silencing strategy employed by mycoviruses (Wang et al., 2016b). To investigate the contributions of RNAi components to the antiviral response against Fusarium graminearum viruses $(\mathrm{FgV1}, 2$, and 3), reverse genetics and virus-derived small RNA profiling were used. FgV1-3 infection differentially induces the gene expression of RNAi components in F. graminearum. FgDICER2 and FgAGO1 transcripts accumulated at lower levels following FgV1 infection than following $\mathrm{FgV} 2$ or $\mathrm{FgV} 3$ infection. FgAGO1 can efficiently robust RNAi response against $\mathrm{FgV1}$ infection, but the functions of two dicer genes, i.e., FgDICER1 and FgDICER2, might be partially redundant in response to $\mathrm{FgV} \mathrm{1-3} \mathrm{infections.}$ These results show that $F$. graminearum developed more complex and robust RNA silencing system against mycoviruses (Yu et al., 2018).

\section{CONCLUSION AND PROSPECTS}

The characterization of the viruses isolated from Fusarium mentioned above has enhanced our understanding of the great diversity of mycoviruses. Most Fusarium mycoviruses have double-stranded or single-stranded, positive- or negativesense RNA genomes. No DNA viruses have been isolated from Fusarium species. Additionally, purified virions of Fusarium mycoviruses can be obtained in large quantities and of high quality and can serve as good material for structural analysis, which will promote a better understanding of mycovirus assembly, function, evolution, and its uses in nanotechnological applications (Ghabrial et al., 2015; De Ruiter et al., 2019).

Fungal viruses causing hypovirulence can be used as potential biocontrol agents of phytopathogenic fungal diseases, which are always restricted by fungal vegetative incompatibility (Son et al., 2015). Recent studies have demonstrated that some mycoviruses can be transmitted between vegetatively incompatible strains, particularly SsHADV-1, Sclerotinia sclerotiorum partitivirus 1 (SsPV1), Sclerotinia sclerotiorum mycoreovirus 4 (SsMYRV4), and Sclerotinia sclerotiorum deltaflexivirus 2 (SsDFV2) (Yu et al., 2013; Xiao et al., 2014; Wu et al., 2017; Hamid et al., 2018). In addition, these mycoviruses can cause hypovirulence. Their hypovirulent properties suggest that SsHADV-1, SsPV1, SsMYRV4, and SsDFV2, have potential for controlling plant disease in the field. Therefore, further research is required to clarify whether the transmission of Fusarium mycoviruses can occur and their corresponding transmission efficiency between vegetative-incompatible individuals or interspecific fungi.

Fusarium graminearum has emerged as a good model system for studying mycovirus-host interactions, similar to three other host-virus interaction systems: C. parasitica-mycovirus, $S$. sclerotiorum-mycovirus, and $R$. necatrix-mycovirus. There are some advantages of using $F$. graminearum-mycovirus for such studies. For example, the genome of the F. graminearum strain $\mathrm{PH}-1$ is sequenced and is available publicly, allowing RNAseq-based, genome-wide expression analysis and modification of targeted gene disruptions. Additionally, protoplasts of F. graminearum are easy to prepare and store and are competent in genetics transformation systems with DNA. Infectious cDNA clones have been successfully used in a few fungal species, including C. parasitica, Diaporthe ambigua, and Sclerotinia sclerotiorum (Choi and Nuss, 1992; Chen et al., 1993, 1994; Chen and Nuss, 1999; Moleleki et al., 2003; Lin et al., 2007; Marzano et al., 2015; Zhang et al., 2016), but not so far in F. graminearum. The 
construction of infectious cDNA clones can be used to explore the properties of mycoviruses and employ mycoviruses as vectors to introduce genes deleterious to the fungal host (Pearson and Bailey, 2013).

Future studies should focus on systematically identifying viral and host factors important for the interactions between mycoviruses and their hosts, especially crucial determinants responsible for the phenotypic changes (including hypovirulence) and reduction of Fusarium mycotoxin production caused by mycovirus infection.

\section{REFERENCES}

Aoki, N., Moriyama, H., Kodama, M., Arie, T., Teraoka, T., and Fukuhara, T. (2009). A novel mycovirus associated with four double-stranded RNAs affects host fungal growth in Alternaria alternata. Virus Res. 140, 179-187. doi: 10.1016/j.virusres.2008.12.003

Bormann, J., Heinze, C., Blum, C., Mentges, M., Brockmann, A., Alder, A., et al. (2018). Expression of a structural protein of the mycovirus FgV-ch9 negatively affects the transcript level of a novel symptom alleviation factor and causes virus infection-like symptoms in Fusarium graminearum. J. Virol. 92, e00326-e00318. doi: 10.1128/JVI.00326-18

Chen, B., Choi, G. H., and Nuss, D. L. (1993). Mitotic stability and nuclear inheritance of integrated viral cDNA in engineered hypovirulent strains of the chestnut blight fungus. EMBO J. 12, 2991-2998. doi: 10.1002/j.1460-2075.1993.tb05967.x

Chen, B., Choi, G. H., and Nuss, D. L. (1994). Attenuation of fungal virulence by synthetic infectious hypovirus transcripts. Science. 264, 1762-1764. doi: $10.1126 /$ science. 8209256

Chen, B., and Nuss, D. L. (1999). Infectious cDNA clone of hypovirus CHV1-Euro7: a comparative virology approach to investigate virus-mediated hypovirulence of the chestnut blight fungus Cryphonectria parasitica. J. Virol. 73, 985-992.

Chen, X., He, H., Yang, X., Zeng, H., Qiu, D., and Guo, L. (2016). The complete genome sequence of a novel Fusarium graminearum RNA virus in a new proposed family within the order Tymovirales. Arch. Virol. 161, 2899-2903. doi: 10.1007/s00705-016-2961-1

Chen, Y., Gao, Q. X., Huang, M. M., Liu, Y., Liu, Z. Y., Liu, X., et al. (2015). Characterization of RNA silencing components in the plant pathogenic fungus Fusarium graminearum. Sci. Rep. 5:13. doi: 10.1038/srep12500

Cho, W. K., Lee, K. M., Yu, J., Son, M., and Kim, K. H. (2013). Insight into mycoviruses infecting Fusarium species. Adv. Virus Res. 86, 273-288. doi: 10.1016/B978-0-12-394315-6.00010-6

Cho, W. K., Yu, J., Lee, K. M., Son, M., Min, K., Lee, Y. W., et al. (2012). Genome-wide expression profiling shows transcriptional reprogramming in Fusarium graminearum by Fusarium graminearum virus 1-DK21 infection. BMC Genomics 13:173. doi: 10.1186/1471-2164-13-173

Choi, G. H., and Nuss, D. L. (1992). Hypovirulence of chestnut blight fungus conferred by an infectious viral cDNA. Science. 257, 800-803. doi: $10.1126 /$ science. 1496400

Chu, Y. M., Jeon, J. J., Yea, S. J., Kim, Y. H., Yun, S. H., Lee, Y. W., et al. (2002). Double-stranded RNA mycovirus from Fusarium graminearum. Appl. Environ. Microbiol. 68, 2529-2534. doi: 10.1128/AEM.68.5.2529-2534.2002

Chu, Y. M., Lim, W. S., Yea, S. J., Cho, J. D., Lee, Y. W., and Kim, K. H. (2004). Complexity of dsRNA mycovirus isolated from Fusarium graminearum. Virus Genes. 28, 135-143. doi: 10.1023/B:VIRU.0000012270.67302.35

Compel, P., Papp, I., Bibo, M., Fekete, C., and Hornok, L. (1999). Genetic interrelationships and genome organization of double-stranded RNA elements of Fusarium poae. Virus Genes 18, 49-56. doi: 10.1023/A:1008069 318838

Darissa, O., Adam, G., and Schäfer, W. (2012). A dsRNA mycovirus causes hypovirulence of Fusarium graminearum to wheat and maize. Eur. J. Plant Pathol. 134, 181-189. doi: 10.1007/s10658-012-9977-5

\section{AUTHOR CONTRIBUTIONS}

SW, LZ, and IA performed the literature search and prepared the manuscript table. LG drafted and revised the manuscript. PL and $\mathrm{PB}$ contributed equally to writing the manuscript. All authors revised and agree with the final manuscript version.

\section{FUNDING}

This work was supported by the National Key R\&D Program of China (2018YFD0200500) to LG.

Darissa, O., Willingmann, P., Schafer, W., and Adam, G. (2011). A novel double-stranded RNA mycovirus from Fusarium graminearum: nucleic acid sequence and genomic structure. Arch. Virol. 156, 647-658. doi: 10.1007/s00705-010-0904-9

Dawe, A. L., and Nuss, D. L. (2013). Hypovirus molecular biology: from Koch's postulates to host self-recognition genes that restrict virus transmission. $A d v$. Virus Res. 86, 109-147. doi: 10.1016/B978-0-12-394315-6.00005-2

De Ruiter, M. V., Klem, R., Luque, D., Cornelissen, J. J. L. M., and Castón, J. R. (2019). Structural nanotechnology: three-dimensional cryo-EM and its use in the development of nanoplatforms for in vitro catalysis. Nanoscale 11, 4130-4146. doi: 10.1039/C8NR09204D

Ding, S. (2010). RNA-based antiviral immunity. Nat. Rev. Immunol. 10, 632-644. doi: $10.1038 /$ nri2824

Ghabrial, S. A., Castón, J. R., Jiang, D., Nibert, M. L., and Suzuki, N. (2015). 50-plus years of fungal viruses. Virology 479-480, 356-368. doi: 10.1016/j.virol.2015.02.034

Ghabrial, S. A., and Suzuki, N. (2009). Viruses of plant pathogenic fungi. Annu. Rev. Phytopathol. 47, 353-384. doi: 10.1146/annurev-phyto-080508-081932

Hamid, M. R., Xie, J., Wu, S., Maria, S. K., Zheng, D., Assane, H. A., et al. (2018). A novel deltaflexivirus that infects the plant fungal pathogen, Sclerotinia sclerotiorum, can be transmitted among host vegetative incompatible strains. Viruses 10:E295. doi: 10.3390/v10060295

Hammond, T. M., Andrewski, M. D., Roossinck, M. J., and Keller, N. P. (2008). Aspergillus mycoviruses are targets and suppressors of RNA silencing. Eukaryot. Cell 7, 350-357. doi: 10.1128/EC.00356-07

He, H., Chen, X., Li, P., Qiu, D., and Guo, L. (2018). Complete genome sequence of a Fusarium graminearum double-stranded RNA virus in a newly proposed family, Alternaviridae. Genome Announc. 6, e00064-e00018. doi: 10.1128/genomeA.00064-18

Hillman, B. I., Annisa, A., and Suzuki, N. (2018). Viruses of plant-interacting fungi. Adv. Virus Res. 100, 99-116. doi: 10.1016/bs.aivir.2017.10.003

Hillman, B. I., Supyani, S., Hideki, K., and Nobuhiro, S. (2004). A reovirus of the fungus Cryphonectria parasitica that is infectious as particles and related to the coltivirus genus of animal pathogens. J. Virol. 78, 892-898. doi: 10.1128/JVI.78.2.892-898.2004

Jiang, D., Fu, Y., Li, G., and Ghabrial, S. A. (2013). Viruses of the plant pathogenic fungus Sclerotinia sclerotiorum. Adv. Virus Res. 86, 215-248. doi: 10.1016/B978-0-12-394315-6.00008-8

Kanhayuwa, L., Kotta Loizou, I., Ozkan, S., Gunning, A. P., and Coutts, R. H. A. (2015). A novel mycovirus from Aspergillus fumigatus contains four unique dsRNAs as its genome and is infectious as dsRNA. Proc. Natl. Acad. Sci. U.S.A. 112, 9100-9105. doi: 10.1073/pnas.1419225112

Kondo, H., Kanematsu, S., and Suzuki, N. (2013). Viruses of the white root rot fungus, Rosellinia necatrix. Adv. Virus Res. 86, 177-214. doi: 10.1016/B978-0-12-394315-6.00007-6

Kozlakidis, Z., Herrero, N., Ozkan, S., Kanhayuwa, L., Jamal, A., Bhatti, M. F., et al. (2013). Sequence determination of a quadripartite dsRNA virus isolated from Aspergillus foetidus. Arch. Virol. 158, 267-272. doi: 10.1007/s00705-012-1362-3

Kwon, S. J., Cho, S. Y., Lee, K. M., Yu, J., Son, M., and Kim, K. H. (2009). Proteomic analysis of fungal host factors differentially expressed by Fusarium graminearum infected with Fusarium graminearum virus-DK21. Virus Res. 144, 96-106. doi: 10.1016/j.virusres.2009.04.004 
Kwon, S. J., Lim, W. S., Park, S. H., Park, M. R., and Kim, K. H. (2007). Molecular characterization of a dsRNA mycovirus, Fusarium graminearum Virus-DK21, which is phylogenetically related to hypoviruses but has a genome organization and gene expression strategy resembling those of plant potex-like viruses. Mol. Cells 28, 73-74. doi: 10.1007/s10059-009-0112-1

Lee Marzano, S. Y., Nelson, B. D., Ajayi-Oyetunde, O., Bradley, C. A., Hughes, T. J., Hartman, G. L., et al. (2016). Identification of diverse mycoviruses through metatranscriptomics characterization of the viromes of five major fungal plant pathogens. J. Virol. 90, 6846-6863. doi: 10.1128/JVI.00357-16

Lee, K. M., Cho, W. K., Yu, J., Son, M., Choi, H., Min, K., et al. (2014). A comparison of transcriptional patterns and mycological phenotypes following infection of Fusarium graminearum by four mycoviruses. PLoS ONE 9:e100989. doi: 10.1371/journal.pone.0100989

Lefkowitz, E. J., Dempsey, D. M., Hendrickson, R. C., Orton, R. J., Siddell, S. G., and Smith, D. B. (2018). Virus taxonomy: the database of the International Committee on Taxonomy of Viruses (ICTV). Nucleic Acids Res. 46, D708D717. doi: 10.1093/nar/gkx932

Lemus-Minor, C. G., Canizares, M. C., Garcia-Pedrajas, M. D., and Perez-Artes, E. (2015). Complete genome sequence of a novel dsRNA mycovirus isolated from the phytopathogenic fungus Fusarium oxysporum f. sp. dianthi. Arch. Virol. 160, 2375-2379. doi: 10.1007/s00705-015-2509-9

Lemus-Minor, C. G., Cañizares-Nolasco, C., Mdd, G. P., and Pérez-Artés, E. (2018). Fusarium oxysporum f. sp. dianthi virus 1 accumulation is correlated with changes in virulence and other phenotypic traits of its fungal host. Phytopathology 108, 957-963. doi: 10.1094/PHYTO-06-17-0200-R

Li, P., Chen, X., He, H., Qiu, D., and Guo, L. (2017). Complete genome sequence of a novel hypovirus from the phytopathogenic fungus Fusarium langsethiae. Genome Announc. 5, e01722-e01716. doi: 10.1128/genomeA.01722-16

Li, P., Lin, Y., Zhang, H., Wang, S., Qiu, D., and Guo, L. (2016). Molecular characterization of a novel mycovirus of the family Tymoviridae isolated from the plant pathogenic fungus Fusarium graminearum. Virology 489, 86-94. doi: 10.1016/j.virol.2015.12.004

Li, P., Zhang, H., Chen, X., Qiu, D., and Guo, L. (2015). Molecular characterization of a novel hypovirus from the plant pathogenic fungus Fusarium graminearum. Virology 481, 151-160. doi: 10.1016/j.virol.2015.02.047

Li, W., Xia, Y., Zhang, H., Zhang, X., and Chen, H. (2019). A Victorivirus from Fusarium asiaticum, the pathogen of Fusarium head blight in China. Arch. Virol. 164, 313-316. doi: 10.1007/s00705-018-4038-9

Lin, H., Lan, X., Liao, H., Parsley TB, Nuss DL, Chen B. (2007). Genome sequence, full-length infectious cDNA clone, and mapping of viral double-stranded RNA accumulation determinant of hypovirus CHV1-EP721. J. Virol. 81, 1813-1820. doi: 10.1128/JVI.01625-06

Liu, L., Xie, J., Cheng, J., Fu, Y., Li, G., Yi, X., et al. (2014). Fungal negative-stranded RNA virus that is related to bornaviruses and nyaviruses. Proc. Natl. Acad. Sci. U.S.A. 111, 12205-12210. doi: 10.1073/pnas.1401786111

Martelli, G. P., Sabanadzovic, S., Sabanadzovic, A. G., Edwards, M. C., and Dreher, T. (2002). The family Tymoviridae. Arch. Virol. 147, 1837-1846. doi: 10.1007/s007050200045

Martínez-Âlvarez, P., Vainio, E. J., Botella, L., Hantula, J., and Diez, J. J. (2014). Three mitovirus strains infecting a single isolate of Fusarium circinatum are the first putative members of the family Narnaviridae detected in a fungus of the genus Fusarium. Arch. Virol. 159, 2153-2155. doi: 10.1007/s00705-014-2012-8

Marvelli, R. A., Hobbs, H. A., Li, S., Mccoppin, N. K., Domier, L. L., Hartman, G. L., et al. (2014). Identification of novel double-stranded RNA mycoviruses of Fusarium virguliforme and evidence of their effects on virulence. Arch. Virol. 159, 349-352. doi: 10.1007/s00705-013-1760-1

Marzano, S.-Y. L., Hobbs, H. A., Nelson, B. D., Hartman, G. L., Eastburn, D. M., Mccoppin, N. K., et al. (2015). Transfection of Sclerotinia sclerotiorum with in vitro transcripts of a naturally occurring interspecific recombinant of Sclerotinia sclerotiorum hypovirus 2 significantly reduces virulence of the fungus. J Virol. 89, 5060-5071. doi: 10.1128/JVI.03199-14

Mizutani, Y., Abraham, A., Uesaka, K., Kondo, H., Suga, H., Suzuki, N., et al. (2018). Novel mitoviruses and a unique tymo-like virus in hypovirulent and virulent strains of the Fusarium head blight fungus, Fusarium boothii. Viruses 10:E584. doi: 10.3390/v10110584

Moleleki, N., Van Heerden, S. W., Wingfield, M. J., Wingfield, B. D., and Preisig, O. (2003). Transfection of Diaporthe perjuncta with Diaporthe RNA virus. Appl. Environ. Microbiol. 69, 3952-3956. doi: 10.1128/AEM.69.7.3952-3956.2003
Munkvold, G. P. (2017). Fusarium species and their associated mycotoxins. Methods Mol. Biol. 1542, 51-106. doi: 10.1007/978-1-4939-6707-0_4

Nogawa, M., Kageyama, T., Nakatani, A. G., Shimosaka, M., and Okazaki, M. (1996). Cloning and characterization of mycovirus double-stranded RNA from the plant pathogenic fungus, Fusarium solani f. sp. robiniae. Biosci. Biotechnol. Biochem. 60, 784-788. doi: 10.1271/bbb.60.784

Nuss, D. L. (2005). Hypovirulence: mycoviruses at the fungal-plant interface. Nat. Rev. Microbiol. 3, 632-642. doi: 10.1038/nrmicro1206

Nuss, D. L. (2011). Mycoviruses, RNA silencing, and viral RNA recombination. Adv. Virus Res. 80, 25-48. doi: 10.1016/B978-0-12-385987-7.00002-6

Osaki, H., Sasaki, A., Nomiyama, K., Sekiguchi, H., Tomioka, K., and Takehara, T. (2015). Isolation and characterization of two mitoviruses and a putative alphapartitivirus from Fusarium spp. Virus Genes 50, 466-473. doi: 10.1007/s11262-015-1182-0

Osaki, H., Sasaki, A., Nomiyama, K., and Tomioka, K. (2016). Multiple virus infection in a single strain of Fusarium poae shown by deep sequencing. Virus Genes 52, 835-847. doi: 10.1007/s11262-016-1379-x

Pearson, M. N., and Bailey, A. M. (2013). Viruses of Botrytis. Adv. Virus Res. 86, 249-272. doi: 10.1016/B978-0-12-394315-6.00009-X

Pearson, M. N., Beever, R. E., Boine, B., and Arthur, K. (2010). Mycoviruses of filamentous fungi and their relevance to plant pathology. Mol. Plant Pathol. 10, 115-128. doi: 10.1111/j.1364-3703.2008.00503.x

Sasaki, A., Kanematsu, S., Onoue, M., Oikawa, Y., Nakamura, H., and Yoshida, K. (2007). Artificial infection of Rosellinia necatrix with purified viral particles of a member of the genus Mycoreovirus reveals its uneven distribution in single colonies. Phytopathology 97, 278-286. doi: 10.1094/PHYTO-97-3-0278

Sharma, M., Guleria, S., Singh, K., Chauhan, A., and Kulshrestha, S. (2018). Mycovirus associated hypovirulence, a potential method for biological control of Fusarium species. Virusdisease 29, 134-140. doi: 10.1007/s13337-01 8-0438-4

Son, H., Park, A. R., Lim, J. Y., Shin, C., and Lee, Y. W. (2017). Genomewide exonic small interference RNA-mediated gene silencing regulates sexual reproduction in the homothallic fungus Fusarium graminearum. PLoS Genet. 13:e1006595. doi: 10.1371/journal.pgen.1006595

Son, M., Choi, H., and Kim, K. H. (2016). Specific binding of Fusarium graminearum Hex1 protein to untranslated regions of the genomic RNA of Fusarium graminearum virus 1 correlates with increased accumulation of both strands of viral RNA. Virology 489, 202-211. doi: 10.1016/j.virol.1 2015.12.013

Son, M., Lee, K.-M., Yu, J., Kang, M., Park, J.-M., Kwon, S.-J., et al. (2013). The HEX1 gene of Fusarium graminearum is required for fungal asexual reproduction and pathogenesis and for efficient viral RNA accumulation of Fusarium graminearum virus 1. J. Virol. 87, 10356-10367. doi: 10.1128/JVI.01026-13

Son, M., Yu, J., and Kim, K. H. (2015). Five questions about mycoviruses. PLoS Pathog. 11:e1005172. doi: 10.1371/journal.ppat.1005172

Stanway, C. A., and Buck, K. W. (1984). Infection of protoplasts of the wheat takeall fungus, Gaeumannomyces graminis var. tritici, with double-stranded RNA viruses. J. Gen. Virol. 65, 2061-2065. doi: 10.1099/0022-1317-65-11-2061

Suzuki, N. (2017). Frontiers in fungal virology. J. Gen. Plant Pathol. 83, 419-423. doi: 10.1007/s10327-017-0740-9

Wang, L., He, H., Wang, S., Chen, X., Qiu, D., Kondo, H., et al. (2018). Evidence for a novel negative-stranded RNA mycovirus isolated from the plant pathogenic fungus Fusarium graminearum. Virology 518, 232-240. doi: 10.1016/j.virol.2018.03.008

Wang, L., Wang, S., Yang, X., Zeng, H., Qiu, D., and Guo, L. (2017). The complete genome sequence of a double-stranded RNA mycovirus from Fusarium graminearum strain HN1. Arch. Virol. 162, 2119-2124. doi: $10.1007 / \mathrm{s} 00705-017-3317-1$

Wang, L., Zhang, J., Zhang, H., Qiu, D., and Guo, L. (2016a). Two novel relative double-stranded RNA mycoviruses infecting Fusarium poae strain SX63. Int. J. Mol. Sci. 17:E641. doi: 10.3390/ijms17050641

Wang, S., Kondo, H., Liu, L., Guo, L., and Qiu, D. (2013). A novel virus in the family Hypoviridae from the plant pathogenic fungus Fusarium graminearum. Virus Res. 174, 69-77. doi: 10.1016/j.virusres.2013.03.002

Wang, S., Li, P., Zhang, J., Qiu, D., and Guo, L. (2016b). Generation of a high resolution map of sRNAs from Fusarium graminearum and analysis of responses to viral infection. Sci. Rep. 6:26151. doi: 10.1038/srep26151 
Wang, S., Zhang, J., Li, P., Qiu, D., and Guo, L. (2016c). Transcriptome-based discovery of Fusarium graminearum stress responses to FgHV1 infection. Int. J. Mol. Sci. 17:E1922. doi: 10.3390/ijms17111922

Wei, S., Bian, R., Andika, I. B., Niu, E., Liu, Q., Kondo, H., et al. (2019). Symptomatic plant viroid infections in phytopathogenic fungi. Proc. Natl. Acad. Sci. U.S.A. 116, 13042-13050. doi: 10.1073/pnas.1900762116

Wu, S., Cheng, J., Fu, Y., Chen, T., Jiang, D., Ghabrial, S. A., et al. (2017). Virus-mediated suppression of host non-self recognition facilitates horizontal transmission of heterologous viruses. PLoS Pathog. 13:e1006234. doi: 10.1371/journal.ppat.1006234

Xiao, X., Cheng, J., Tang, J., Fu, Y., Jiang, D., Baker, T. S., et al. (2014). A novel partitivirus that confers hypovirulence on plant pathogenic fungi. J. Virol. 88, 10120-10133. doi: 10.1128/JVI.01036-14

Xie, J., and Jiang, D. (2014). New insights into mycoviruses and exploration for the biological control of crop fungal diseases. Annu. Rev. Phytopathol. 52, 45-68. doi: 10.1146/annurev-phyto-102313-050222

Yu, J., Kwon, S. J., Lee, K. M., Son, M., and Kim, K. H. (2009). Complete nucleotide sequence of double-stranded RNA viruses from Fusarium graminearum strain DK3. Arch. Virol. 154, 1855-1858. doi: 10.1007/s00705-009-0507-5

Yu, J., Lee, K. M., Cho, W. K., Park, J. Y., and Kim, K. H. (2018). Differential contribution of RNA interference components in response to distinct Fusarium graminearum virus infections. J. Virol. 92, e01756-e01717. doi: 10.1128/JVI.01756-17

Yu, J., Lee, K. M., Son, M., and Kim, K. H. (2015). Effects of the deletion and over-expression of Fusarium graminearum gene FgHal2 on host response to mycovirus Fusarium graminearum virus 1. Mol. Plant Pathol. 16, 641-652. doi: $10.1111 / \mathrm{mpp} .12221$

Yu, J.-S., Lee, K.-M., Son, M.-I., and Kim, K.-H. (2011). Molecular characterization of Fusarium graminearum virus 2 Isolated from Fusarium graminearum strain 98-8-60. Plant Pathol. J. 27, 285-290. doi: 10.5423/PPJ.2011.27.3.285
Yu, X., Li, B., Fu, Y., Xie, J., Cheng, J., Ghabrial, S. A., et al. (2013). Extracellular transmission of a DNA mycovirus and its use as a natural fungicide. Proc. Natl. Acad. Sci. U.S.A. 110, 1452-1457. doi: 10.1073/pnas.1213755110

Zhang, R., Hisano, S., Tani, A., Kondo, H., Kanematsu, S., and Suzuki, N. (2016). A capsidless ssRNA virus hosted by an unrelated dsRNA virus. Nat. Microbiol. 1:15001. doi: 10.1038/nmicrobiol.2015.1

Zhang, R., Liu, S., Chiba, S., Kondo, H., Kanematsu, S., and Suzuki, N. (2014). A novel single-stranded RNA virus isolated from a phytopathogenic filamentous fungus, Rosellinia necatrix, with similarity to hypo-like viruses. Front. Microbiol. 5:360. doi: 10.3389/fmicb.2014.00360

Zhang, X., Gao, F., Zhang, F., Xie, Y., Zhou, L., Yuan, H., et al. (2018). The complete genomic sequence of a novel megabirnavirus from Fusarium pseudograminearum, the causal agent of wheat crown rot. Arch. Virol. 163, 3173-3175. doi: 10.1007/s00705-018-3970-Z

Zhang, X., Xie, Y., Zhang, F., Sun, H., Zhai, Y., Zhang, S., et al. (2019). Complete genome sequence of an alternavirus from the phytopathogenic fungus Fusarium incarnatum. Arch. Virol. 164, 923-925. doi: 10.1007/s00705-018-04128-2

Conflict of Interest Statement: The authors declare that the research was conducted in the absence of any commercial or financial relationships that could be construed as a potential conflict of interest.

Copyright (C) 2019 Li, Bhattacharjee, Wang, Zhang, Ahmed and Guo. This is an open-access article distributed under the terms of the Creative Commons Attribution License (CC BY). The use, distribution or reproduction in other forums is permitted, provided the original author(s) and the copyright owner(s) are credited and that the original publication in this journal is cited, in accordance with accepted academic practice. No use, distribution or reproduction is permitted which does not comply with these terms. 